\title{
ARTICLE \\ Low-dose intranasal oxytocin delivered with Breath Powered device modulates pupil diameter and amygdala activity: a randomized controlled pupillometry and fMRI study
}

\author{
Daniel S. Quintana (D) ${ }^{1}$, Lars T. Westlye $\mathbb{D}^{1,2}$, Dag Alnæs (D) ${ }^{1}$, Tobias Kaufmann ${ }^{1}$, Ramy A. Mahmoud ${ }^{3}$, Knut T. Smerud ${ }^{4}$, \\ Per G. Djupesland ${ }^{5}$ and Ole A. Andreassen ${ }^{1}$
}

\begin{abstract}
Little is known about how intranasally administered oxytocin reaches the brain and modulates social behavior and cognition. Pupil dilation is a sensitive index of attentional allocation and effort, and inter-individual variability in pupil diameter during performance of social-cognitive tasks may provide a better assessment of pharmacological effects on the brain than behavioral measures. Here, we leverage the close relationship between pupil and neural activity to inform our understanding of nose-to-brain oxytocin routes and possible dose-response relationships. To this end, we assessed pupil diameter data from a previously reported functional magnetic resonance imaging (fMRI) study under four treatment conditions-including two different doses of intranasal oxytocin using a novel Breath Powered nasal device, intravenous (IV) oxytocin, and placebo-and investigated the association with amygdala activation in response to emotional stimuli. The study used a randomized, double-blind, double-dummy, crossover design, with 16 healthy male adults administering a single-dose of these four treatments. A significant main effect of treatment condition on pupil diameter was observed. Posthoc tests revealed reduced pupil diameter after 8IU intranasal oxytocin compared to placebo, but no significant difference between $8 \mathrm{IU}$ intranasal oxytocin and either 24IU intranasal oxytocin or IV oxytocin treatment conditions. Analysis also showed a significant relationship between pupil diameter and right amygdala activation after $8 \mathrm{IU}$ intranasal oxytocin. Although there was no significant difference between 8IU intranasal oxytocin and IV oxytocin on right amygdala activity and pupil diameter, the significant difference between $8 I U$ intranasal oxytocin and placebo is consistent with the hypothesis that oxytocin can travel to the brain via a nose-to-brain route.
\end{abstract}

Neuropsychopharmacology (2019) 44:306-313; https://doi.org/10.1038/s41386-018-0241-3

\section{INTRODUCTION}

The neuropeptide oxytocin has attracted considerable lay and scientific interest for its potential to address social dysfunction in psychiatric illness. For instance, it has been shown to improve social cognition [1-3], enhance memory for social information [4], and increase gaze to the eye region [5]. Chronic administration in children with autism spectrum disorders has also been found to improve caregiver-rated social functioning [6], pointing to its promise as a psychotherapeutic agent [7]. However, the hype surrounding oxytocin has birthed lofty expectations, with a number of disappointing results tempering early enthusiasm [8].

With accumulating oxytocin studies, researchers are better understanding the conditions under which oxytocin exerts its effects and adjusting their expectations accordingly. For instance, during the early stages of human biobehavioral oxytocin research, it was originally described as a pro-social hormone [9-11]. This likely contributed to its lay popularization as the "cuddle chemical" (e.g., ref. [12]). However, with new evidence revealing that oxytocin also facilitates non-prosocial behaviors [13, 14], researchers have reevaluated their understanding of oxytocin. In light of this new evidence two models of oxytocin have been gaining popularity: the "social salience" and "social-approach/ withdrawal" models. The social salience model suggests oxytocin enhances the salience of social cues [15], while the socialapproach/withdrawal model suggests that oxytocin increases social-approach-related behaviors and reduces withdrawalrelated behaviors [16]. These approach-related behaviors are associated with movement towards appetitive goals, (e.g., emotional engagement) and are not necessarily pro-social (e.g., anger; [17]), whereas withdrawal-related behaviors are associated with the avoidance of aversive stimuli (e.g., fear).

Neurobiological markers have been used to test the social salience and social-approach/withdrawal models of oxytocin for the purposes of better understanding oxytocin's social-behavioral effects. Given the established relationship between cognitive resource allocation and pupil dilation [18, 19], pupillometry offers a non-invasive neurobiological measure of processing cognitive load [20-23]. Intranasal oxytocin administration has been reported to enhance pupil dilation (i.e., increased attentional resources) during the presentation of emotional stimuli [24, 25], which is consistent with the social salience hypothesis. The central release of oxytocin via vaginocervical mechano-stimulation in rats [26] has

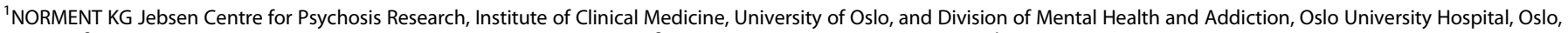

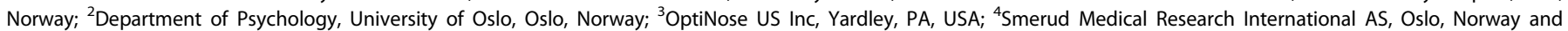
${ }^{5}$ OptiNose AS, Oslo, Norway

Correspondence: Ole A. Andreassen (o.a.andreassen@medisin.uio.no)

Received: 22 March 2018 Revised: 27 September 2018 Accepted: 1 October 2018

Published online: 16 October 2018 
been shown to dilate pupils, and this effect is attenuated with an oxytocin antagonist [27]. In parallel with pupillometry research, fMRI has also been used to understand the neural effect of intranasal oxytocin administration. Studies have repeatedly demonstrated that oxytocin administration reduces amygdala activity during the processing of emotional stimuli-at least in males-regardless of emotional valence (e.g., refs. [28-31]). Moreover, the amygdala has been shown to be involved in relevance detection, in line with social salience function [32]. However, such results from brain imaging studies are at odds with the social salience hypothesis [33], as one would expect enhanced social salience to be associated with increased amygdala activity, compared to placebo, when contrasting emotional stimuli with neutral stimuli $[16,28]$. This $\mathrm{fMRI}$ research is also inconsistent with the pupillometry literature given that amygdala stimulation induces pupil dilation [34, 35]. Direct administration of oxytocin and arginine vasopressin into the eye has also been reported to constrict the rabbit pupil, both in vitro and in vivo, suggesting that oxytocin may act directly on iris sphincter muscles [36]. Intranasal oxytocin administration in dogs was shown to constrict the pupils in response to the presentation of angry human faces compared to happy human faces, however, the opposite effect was found after placebo administration [37]. Of note, prior human pupillometry research has not included non-social stimuli, so it is unclear if the effects of oxytocin on pupil activity are exclusive to social stimuli or if they also modulate pupil activity during the processing of non-social stimuli.

In addition to these conflicting neurobiological effects, the manner in which intranasally administered oxytocin reaches the brain to exert its effects - or if it even reaches the brain at all-is unclear [38]. There is some human evidence that intranasal oxytocin administration increases central concentrations of oxytocin [39], however, it is unknown if this increase is due to direct nose-to-brain transport or through delivery across the blood brain barrier (BBB) via peripherally circulating blood. We have previously shown that an $8 \mathrm{IU}$ dose of oxytocin modulates social cognition [40] and amygdala activity, and that IV oxytocin administration (despite eliciting similar peripheral concentrations) does not elicit effects, which is consistent with nose-to-brain transport of oxytocin with intranasal administration [31, 41]. However, the dose- and route-response effects on pupil activity, and its relationship with brain activity, are uncertain.

To better characterize the role of stimuli engagement in oxytocin response and reconcile disparate pupillometry and fMRI outcomes, here we report the pupillometry findings from a previously reported randomized, double-blind, double-dummy, 4way crossover study in healthy volunteers [31, 41]. By integrating novel pupillometry data and re-examining previously reported fMRI data, we investigated how pupil activity is associated with oxytocin treatment and amygdala activity during the processing of social and non-social stimuli. We have shown that "low dose" (8IU) OT delivered with a Breath Powered OptiNose device (OPNOT) is more efficient than "higher dose" (24IU) OPN-OT, OT delivered intravenously (IV; 1IU), and placebo [41], but the effects of these dosages and administration routes on pupil activity are not known. The relationships between mean pupil diameter, amygdala activation during the presentation of faces and emotional ratings were also assessed.

\section{MATERIALS AND METHODS}

Participants

The current study is based on data from the study described in detail in previous publications [31, 41]. Participants were recruited through advertisements at the University of Oslo, and males aged 18 to 35 (inclusive) in good physical and mental health were eligible. Exclusion criteria included use of any medications within the last 14 days, history of alcohol or drug abuse, and $\mathrm{IQ}<75$. A screening visit occurred between 3-21 days prior to randomization at Oslo University Hospital. The Wechsler Abbreviated Scale of Intelligence [42] and the Mini-International Neuropsychiatric Interview [43] were administered by trained graduate students under the supervision of study physicians and clinical psychologists to index IQ and confirm the absence of psychiatric illness, respectively. A physical examination including ECG and routine blood sampling was performed by study physicians and nurses. An otolaryngologist confirmed normal nasal anatomy and patency in participants via physical examination consistent with recent recommendations [44] and acoustic rhinometry (AR) data were collected by trained study staff under the supervision of an otolaryngologist (SRE 2000; Rhinometrics, A/S, Smørum, Denmark). This study was approved by the Regional Committee for Medical and Health Research Ethics (REC South East) and carried out in compliance with the latest revision of the Declaration of Helsinki. Participants provided written informed consent before they participated (see also ref. [41]). The study was registered at the U.S. National Institutes of Health clinical trial registry (www. clinicaltrials.gov; NCT01983514) and as EudraCT no. 2013-00160812.

Fifty-seven male volunteers were assessed for eligibility, and 18 participants aged 20-30 years $(M=23.81, S D=3.33)$ were randomized. On average, 8 days elapsed between each treatment session (range: 6-20 days, $\mathrm{SD}=3.5$ days). Two participants withdrew after enrollment [1 withdrew after the first session (Placebo) and the other withdrew after completing three sessions (8IU OPN-OT, IV-OT, Placebo)]. Data from these participants are not included in the analyses. Recruitment commenced September 2013 and the last data were collected February 2014.

\section{Study design}

Participants received 8IU OPN-OT intranasally, 24IU OPN-OT intranasally, 1IU OT delivered intravenously, and placebo in a randomized, placebo-controlled, double-blind, double-dummy, four-period crossover design. Both participants and research team were blind to treatment randomization, with the study contract research organization (Smerud Medical Research International, Oslo, Norway) performing the randomization. For study design details, see refs. [31, 41]. All participants self-administered an intranasal treatment using the Breath Powered device and also received an IV solution in all treatment periods. The contents of the nasal spray and IV solutions (oxytocin or placebo) depended on randomization (8IU condition: 8IU nasal spray and placebo IV; 24IU condition: 24IU nasal spray and placebo IV; IV condition: placebo nasal spray and 1IU IV OT; Placebo condition: placebo nasal spray and placebo IV). A pragmatic approach was taken for sample size determination reflecting the phase 1 status of OT administration using the Breath Powered device and the complex nature of the study design.

\section{Breath Powered delivery device and OT administration}

A novel approach to intranasal drug delivery, sometimes referred to as "Breath Powered" was used for oxytocin administration in this study [31,41]. This approach uses what is formally referred to as an exhalation delivery system (EDS) that exploits the natural physiology of exhalation against resistance, including a sealed soft palate, to deliver medication. Exhalation delivery systems have been shown to deposit drug more superiorly and posteriorly than standard pump-actuated nasal sprays [45]. To date, two products using an EDS (for other molecules) have been approved by the U.S. FDA. In this study, the Breath Powered device was fitted with a slightly elongated nosepiece with a sideways flexible tip to further optimize delivery to the most upper and posterior segments of the nasal cavity $[41,46]$. AR was performed prior to treatment administration to confirm that the nasal valve dimensions did not significantly differ between sessions [47]. 
Study tasks and data acquisition

The social cognition task and fMRI sequence began 40 min after intranasal administration, lasting $21 \mathrm{~min}$. Participants were presented with visual stimuli through MRI-compatible goggles (VisualSystem; NordicNeuroLab AS, Bergen, Norway) using EPrime 2.0 (Psychology Software Tools Inc, Sharpsburg, PA, USA) and responded using a grip response collection system (ResponseGrip, NordicNeuroLab AS, Bergen, Norway). In an event-related design, participants were presented with 20 male and 20 female faces (as used previously; $[24,41]$ ) displaying angry, happy and emotionally ambiguous facial expressions (derived from the Karolinska Directed Emotional Faces database; [48]) and 20 images of geometrical shapes. For details, see previously published research $[31,41]$.

MRI data was collected on a $3 \mathrm{~T}$ General Electric Signa HDxt scanner with an 8-channel head coil (GE Healthcare, Milwaukee, WI, USA). The protocol included a $\mathrm{T}^{*}$-weighted gradient echoplanar imaging (EPI) sequence acquired in the transverse plane with the following parameters: Repetition time $(T R)=2400 \mathrm{~ms}$, echo time $(T E)=30 \mathrm{~ms}$, flip angle $(F A)=90^{\circ}, 64 \times 64$ matrix. One run of 528 volumes was collected for each individual in each OT condition (48 slices; in-plane resolution $3.75 \times 3.75 \mathrm{~mm}$; slice thickness $3.2 \mathrm{~mm}$, no gap). A T1-weighted volume used for coregistration purposes was acquired using a sagittal fast spoiled gradient echo (FSPGR) sequence with the following parameters: $\mathrm{TR}=7.8 \mathrm{~ms}, \mathrm{TE}=2.9 \mathrm{~ms}$, flip angle $=12^{\circ}, 166$ slices; in-plane resolution: $1 \times 1$, slice thickness: $1.2 \mathrm{~mm}, 256 \times 256$ matrix.

Pupillometry data was collected using an MR-compatible coilmounted infrared EyeTracking system (NNL EyeTracking camera ${ }^{\circledR}$, NordicNeuroLab AS, Bergen, Norway) at a sampling rate of $60 \mathrm{~Hz}$. Data was recorded using the iView $X$ Software (SensoMotoric Instruments, Teltow, Germany), with a trigger from the stimulus computer synchronizing the onset of the pupillometry recording to stimulus presentations.

Data processing and analysis

FreeSurfer (http://surfer.nmr.mgh.harvard.edu) was used for the T1-weighted data, including surface reconstruction and full brain segmentation [49] to obtain precise brain extracted volumes for co-registration of the fMRI data. FMRIB Software Library (FSL; http://fsl.fmrib.ox.ac.uk/fsl/fslwiki/; [50]) was used to process fMRI data. The first five volumes were discarded. Pre-processing of fMRI data was conducted using FMRIB's Expert Analysis Tool (FEAT) version 6.0 [51]. This included motion correction using MCFLIRT [50], spatial smoothing by means of SUSAN [52] using a Gaussian kernel of FWHM of $7 \mathrm{~mm}$, and a temporal high pass filter of $100 \mathrm{~s}$. FMRIB's Linear and non-linear Image Registration Tools (FLIRT; [50]) optimized using Boundary-Based Registration (BBR; [53]) was used to align each participant's fMRI data to a standard space (MNI-152) with the T1-weighted volume as an intermediate.

We fitted individual level general linear models (GLM) using FILM (FMRIB's Improved Linear Model; [51, 54]) modeling the facial stimuli (happy/angry/ambiguous faces) and geometrical shape and fixations as events and interspersed fixations and pause periods, Q1 and Q2 were modeled as regressors across the different facial stimuli and shapes. Next, we extracted the average amygdala contrast-parameter estimates (COPE) from left and right amygdala masks based on the Harvard-Oxford anatomical atlas provided with FSL and submitted the values to higher-level analysis (see below). We also extracted COPE values from left and right amygdala masks from the contrast comparing emotional faces and shapes.

Pupillometry data were pre-processed using a custom-made MATLAB (MathWorks Inc., Natick, MA, USA) script (available upon request). Raw data were converted into diameters, with physiologically unlikely pupil sizes ( $<2 \mathrm{~mm}$ or $>9 \mathrm{~mm}$ ) excluded from the data to remove noise (e.g., eye blinks). To assess the association with amygdala activity during the presentation of

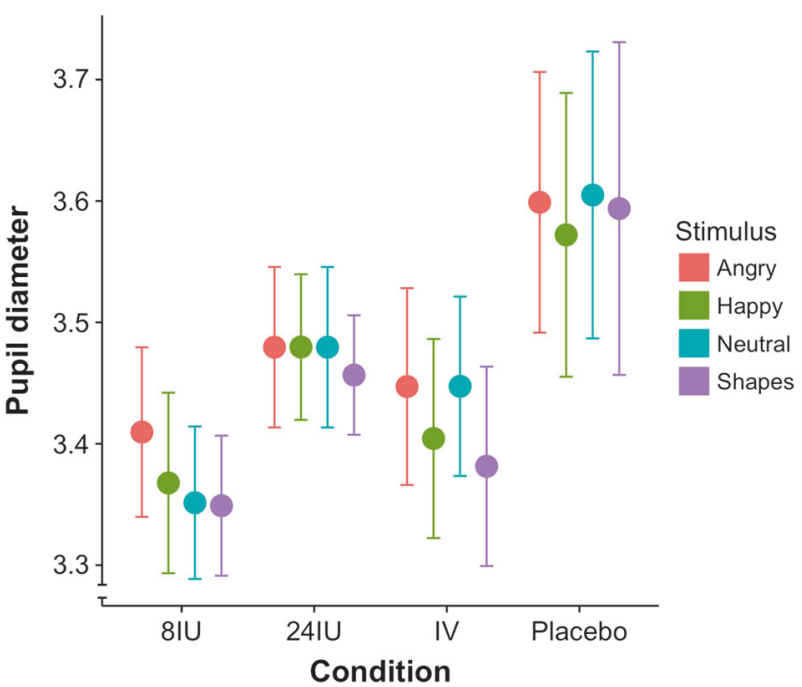

Fig. 1 Main effects and interactions of treatment and stimulus type on pupil diameter. Means and standard errors are shown for pupil diameter (millimeters) while processing presented stimuli after each treatment

stimuli, each time series was split into epochs of $8 \mathrm{~s}$ duration starting at stimulus presentation. For each treatment session, we calculated the average pupil diameter in each epoch and then across all 20 epochs per stimulus condition to generate mean overall pupil diameters per stimulus condition (angry face, happy face, neutral face, geometric shape).

Statistical analysis was conducted using $\mathrm{R}$ [55]. Linear mixedeffects models (LMM) were fitted using the "nlme" package (http://CRAN.R-project.org/package $=$ nlme) to first evaluate the main effects of stimulus category (face vs. shape) and emotion type (happy vs. angry vs. neutral) on pupil diameter, and then the main effects of treatment condition (8IU oxytocin, 24IU oxytocin, IV oxytocin, placebo), stimulus type (happy faces, angry faces, neutral faces, shapes), and their interaction on pupil diameter and the percentage of data removed due to artifacts, such as blinking. $P$-values from traditional frequentist inference cannot be used to provide evidence for a null-hypothesis, no matter how large the $P$ value [56]. Alternatively, Bayesian inference can be used to assess the relative evidence of a null model to an alternative model (for an accessible introduction to Bayesian inference, see $[57,58])$. Therefore, Bayesian mixed models were used to complement frequentist inference by examining the relative strength of evidence for both the null and alternative hypotheses using the Jeffreys-Zellner-Siow (JZS) prior [59]. A Bayes factor (BF) value $<0.33$ suggests that the null model is three times more favored than the alternative model, given the data. A BF over 3 suggests that the alternative model is three times more favored than the null model, given the data [60]. BFs can also provide a measure of data sensitivity, in other words, whether the sample size was large enough to support one hypothesis over another [61]. BFs between 0.33 and 3 may indicate that more participants are required, whereas BFs $<0.33$ or $>3$ suggest that the sample size was sufficient to provide relative evidence for either the null or alternative hypotheses [61]. Frequentist correlation coefficients and Bayesian (JZS prior) correlations were also calculated to assess the relationship between mean pupil diameters and previously reported cognitive and amygdala activity data [31, 41].

\section{RESULTS}

Experimental effects on pupil diameter

There was no significant main effect of stimulus category [face vs. geometric shape; $F(1,239)=0.26, P=0.61 ; \mathrm{BF}=0.18$ ] or emotion 


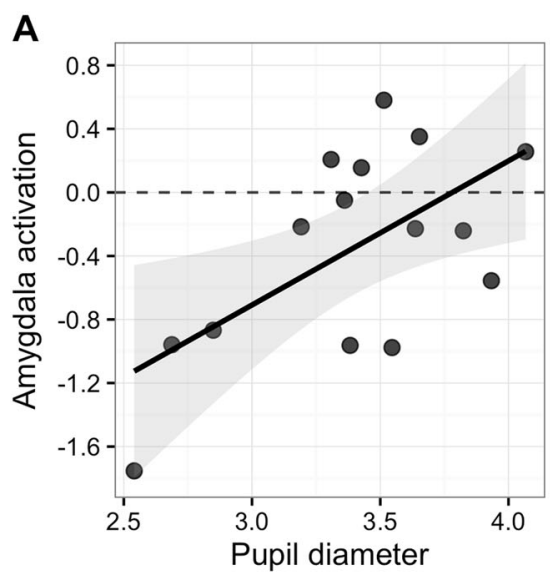

C
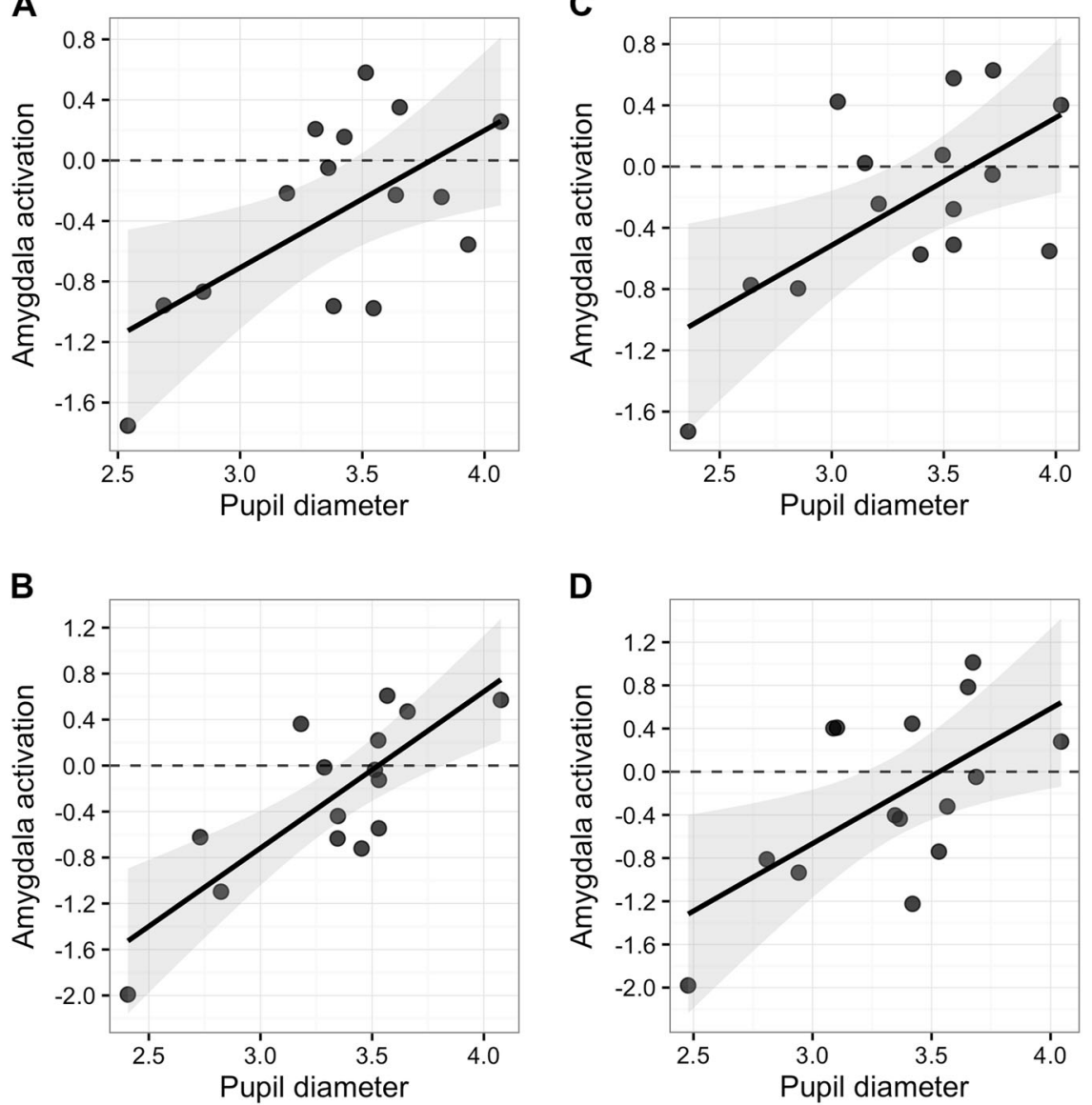

Fig. 2 The relationship between mean pupil diameter and right amygdala activity after 8IU OPT-OT. The significant positive relationship indicates that increased right amygdala activation (z-score normalized) is associated with increased mean pupil diameter while processing angry faces (a), ambiguous faces (b), happy faces (c), and shape stimuli (d) after 8IU OPT-OT. Line of best fit shown with $95 \%$ confidence band. The dashed horizontal line illustrates a normalized $z$-score of 0

\begin{tabular}{|c|c|c|c|c|c|c|c|c|c|c|c|}
\hline \multicolumn{3}{|l|}{ 8IU OPN-OT ${ }^{a}$} & \multicolumn{3}{|l|}{$24 I U$ OPN-OT } & \multicolumn{3}{|l|}{$\mathrm{IV} \mathrm{OT}^{\mathrm{b}}$} & \multicolumn{3}{|l|}{ Placebo $^{b}$} \\
\hline$r(95 \% \mathrm{Cl})$ & $P$ & $\mathrm{BF}$ & $r(95 \% \mathrm{Cl})$ & $P$ & $\mathrm{BF}$ & $r(95 \% \mathrm{Cl})$ & $P$ & $\mathrm{BF}$ & $r(95 \% \mathrm{Cl})$ & $P$ & BF \\
\hline $.61(.14, .86)$ & .02 & 3.66 & $.08(-.43, .55)$ & 0.77 & .2 & $-.22(-.65, .31)$ & 0.42 & .26 & $.24(-.29, .65)$ & .38 & .28 \\
\hline $.79(.47, .93)$ & $<.001$ & 89.9 & $-.04(-.52, .47)$ & 0.89 & .2 & $-.11(-.58, .41)$ & .68 & .26 & $.07(-.44, .54)$ & .81 & .28 \\
\hline $.63(.17, .86)$ & .01 & 4.35 & $.06(-.45, .54)$ & 0.82 & .19 & $-.18(-.62, .35)$ & .5 & .24 & $.22(-.31, .64)$ & .42 & .26 \\
\hline
\end{tabular}

type [happy vs. angry vs. neutral; $F(2,174)=0.11, P=0.9 ; \mathrm{BF}=$ 0.06 ] on mean pupil diameter.

Treatment effects on pupil diameter

There was a significant main effect of treatment condition on mean pupil diameter across all stimuli types $[F(3,225)=5.06$, $P=0.002$; Fig. 1]. The BF was 15 , indicating that the alternative model was 15 times more likely than the null model. Posthoc $t$-test comparisons (Holm corrected for multiple tests) revealed that pupil diameter was significantly smaller after $8 I U$ oxytocin compared to placebo $(P=0.03)$. There were no other statistically significant differences in pupil diameters between treatment conditions. There was no main effect of stimulus type $[F(3,225)=$
0.16, $P=0.923 ; \quad B F=0.02]$, or treatment $\times$ stimulus condition interaction $[F(9,225)=0.05, P=0.999 ; \mathrm{BF}=0.01]$. In regards to the percentage of artifacts removed from the pupillometry data, there was also no main effect of treatment condition $[F(3,225)=$ $1.82, P=0.14 ; \mathrm{BF}=0.21]$, stimulus type $[F(3,225)=0.14, P=0.93$; $\mathrm{BF}=0.03]$, or treatment condition $\times$ stimulus type interaction $[F(9$, 225) $=0.03, P=0.99 ; \mathrm{BF}=0.01]$.

Associations between amygdala fMRI activation and pupil diameter

There was a significant relationship between mean right amygdala activation and mean pupil diameter during the presentation of angry faces $(P=0.02 ; \mathrm{BF}=3.66$; Fig. $2 \mathrm{a})$, ambiguous faces 
Table 2. Relationship between pupil diameter and left amygdala activation after each treatment

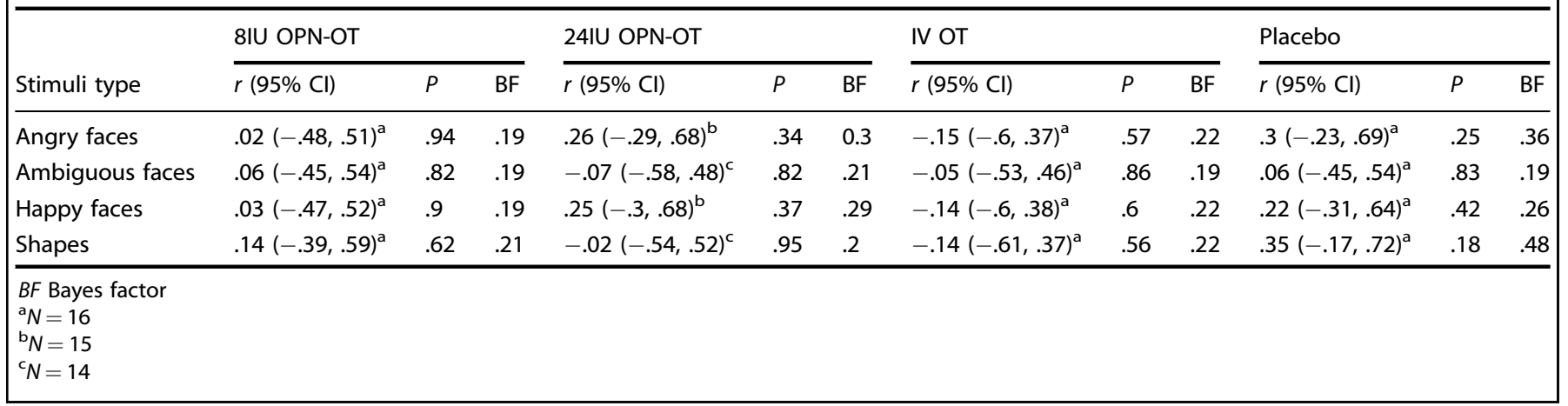

Table 3. Relationship between pupil diameter and emotion ratings after each treatment

\begin{tabular}{|c|c|c|c|c|c|c|c|c|c|c|c|c|}
\hline & \multicolumn{3}{|l|}{ 8IU OPN-OT } & \multicolumn{3}{|l|}{$24 I U$ OPN-OT } & \multicolumn{3}{|l|}{ IV OT $^{\mathrm{b}}$} & \multicolumn{3}{|l|}{ Placebo $^{\text {b }}$} \\
\hline Angry ratings-Angry faces & $-.02(-.53, .49)$ & .92 & .2 & $-.24(-.64, .31)$ & .42 & .26 & $.47(-.05, .79)$ & .07 & .95 & $-.53(-.81,-.05)$ & .03 & 1.75 \\
\hline Angry ratings-Neutral faces & $.16(-.39, .62)$ & .57 & .2 & $.2(-.33, .63)$ & .46 & .25 & $.1(-.44, .58)$ & .73 & .21 & $-.08(-.55, .43)$ & .77 & .2 \\
\hline Happy ratings-Happy faces & $.07(-.46, .56)$ & .8 & .2 & $-.1(-.57, .41)$ & .71 & .2 & $.15(-.39, .62)$ & .59 & .22 & $-.18(-.62, .35)$ & .51 & .23 \\
\hline Happy ratings-Neutral faces & $-.1(-.58, .43)$ & .72 & .21 & $-.01(-.5, .5)$ & .99 & .19 & $-.05(-.54, .48)$ & .87 & .2 & $-.15(-.6, .37)$ & .58 & .22 \\
\hline
\end{tabular}

$(P<0.001 ; \mathrm{BF}=89.9 ;$ Fig. $2 \mathrm{~b})$, happy faces $(P=0.01 ; \mathrm{BF}=4.35$; Fig. 2c), and shapes $(P=0.02 ; B F=3.58 ;$ Fig. $2 \mathrm{~d})$, after $8 I U$ OPN-OT treatment (Table 1). As all the corresponding BFs were $>3$ this provides substantial evidence [60] that the respective variables were related. There were no significant relationships after the other treatments (all $P^{\prime} s>0.05$; Table 1, Fig. S1), and all BFs were less than 0.33 , providing substantial evidence that none of these variables were related. As the other treatments (24IU intranasal oxytocin, IV oxytocin, placebo) included data from 16 participants, whereas the 8IU condition included data from 15 participants, we performed a "leave-one-out" analysis whereby we iteratively removed one participant from the analysis and re-calculated the correlations for each treatment and stimuli condition. None of these correlations were statistically significant, suggesting that that additional scan did not affect statistical significance (Table S1). There was no significant association between left amygdala activity and pupil diameter (Table 2). There were also no statistically significant associations between mean pupil diameter and COPE values from the contrast comparing emotional faces and shapes for the right (Table S2) or left (Table S3) amygdala.

Emotional ratings

There were no statistically significant relationships between intensity of anger or happy ratings and mean pupil diameter (Table 3). Moreover, all the BFs (except for two; Table 3) provided substantial evidence that these variables were not related.

\section{DISCUSSION}

In this double-blind, placebo-controlled crossover study in healthy volunteers, we show that compared to placebo, 8IU oxytocin significantly reduced pupil diameter recorded during presentation of visual stimuli. We also demonstrate a significant association between right amygdala activation and mean pupil diameter during the presentation of stimuli after 8IU oxytocin. Both observations occurred with positively and negatively valenced social stimuli (angry, ambiguous, and happy faces) and non-social stimuli (geometric shapes). Given that pupil dilation reflects cognitive load, these results provide support for the socialapproach oxytocin hypothesis, rather than the social salience hypothesis. Taken together, the current neurobiological data suggest that low-dose OPN-OT, but not high-dose OPN-OT, restrains the marshaling of cognitive resources for attending to stimuli, regardless of valence or social characteristics.

We did not observe such inhibitory effects after 24IU intranasal oxytocin or $1 \mathrm{IU}$ intravenous oxytocin compared to placebo. The lack of effects after 1IU intravenous oxytocin is in line with the hypothesis that intranasal oxytocin administration delivers oxytocin to the brain via nose-to-brain nerve fiber pathways rather than across the BBB via peripheral circulation [62]. However, it should be noted that there was no statistically significant difference in pupil diameter between 8IU intranasal oxytocin and $1 \mathrm{IU}$ intravenous oxytocin. Despite the observed main effects of treatment on pupil diameter, we previously reported no main effects on self-reported anxiety [41], indicating that changes in pupil diameter were not related to any overt anxiolytic effects.

The data also suggest that after low dose 8IU oxytocin there is a positive relationship between pupil diameter and right amygdala activation when processing faces and shapes. This is consistent with findings suggesting that decreased amygdala activity is associated with pupil constriction [34, 35]. As amygdala activity is associated with the presentation of both negative and positive unfamiliar stimuli [63] and pupil diameter reflects the cognitive resource allocation [21], oxytocin may facilitate both social and non-social-approach-related behaviors by reducing uncertainty and vigilance towards novel stimuli $[28,33,64]$. Altogether, these results suggest that oxytocin exerts a more general effect on stimuli processing that may not be specific to social stimuli, in line with the previously reported function of the amygdala [32]. It is not immediately clear why we did not observe a relationship between left amygdala activity and pupil constriction. Indeed, there is a mixed literature on the laterality of the effects of 
intranasal oxytocin on the attenuation of amygdala activity [65]. Our previously reported results using the same dataset revealed that 8IU intranasal oxytocin only attenuated activity in the right amygdala [31]. Similarly, Domes and colleagues ([28]) reported reduced amygdala activation in response to the presentation of both angry and happy faces. It has been suggested that the right amygdala underlies the immediate response to visual stimuli [6668], which may explain why only a significant relationship between right amygdala activity and pupil diameter was found in response to social and non-social stimuli.

The behavioral and neuronal effects of oxytocin have mainly been studied using paradigms probing social-cognitive processes and contexts. However, the present results indicate that the pupil response to intranasal oxytocin may not necessarily be unique to social stimuli, as there was no significant relationship between pupil diameter and amygdala activation in the contrast comparing faces and shapes. Moreover, we have previously reported using the same dataset that there was no main effect of treatment on amygdala response to faces compared to shapes [31], corroborating that the effect of intranasal oxytocin on amygdala activity may not be unique to social stimuli. Considering that oxytocin influences a variety of homeostatic processes [69], it would be somewhat surprising if neurophysiological responses to endogenous oxytocin were exclusive to social stimuli. Indeed, the oxytocin system has been shown to influence a range of non-social animal behaviors including learning [70], location preference [71], and pain tolerance [72]. Human research has also demonstrated that oxytocin administration reduces amygdala activation during pain stimulation [73] and decreases trait-based rule adherence [74]. Despite that fact we did not collect data explicitly reflecting approach-related behaviors, the data in the current study is consistent with increasing evidence that oxytocin increases both social and non-social-approach-related behaviors [64].

By comparing pupillometry and amygdala data, this study adopted a multimodal approach to understanding the biological underpinnings of the response to intranasal oxytocin. Moreover, this approach was well-suited to reconcile conflicting pupillometry and fMRI results, insofar that reduced amygdala activity would be expected to be associated with pupil constriction. While the pupillometry results appear to be inconsistent with prior work $[24,25]$, it is important to note that these divergent results may reflect the difficulty of the present task. The task in the present experiment was relatively easy, with participants asked to rate levels of happiness and anger on a Likert scale. Previous oxytocin pupillometry studies included more complex social cognition tasks requiring sustained attention, such as recognizing emotions in morphing faces [25]. Also, these inconsistent results may be due to measurement of different aspects of the pupillary response. For instance, in the study by Leknes and colleagues ([24]), the pupil response for a stimulus was assessed by measuring change from a pre-stimulus mean diameter. We measured pupil diameter over a prolonged period (i.e., a block) and thus our measure cannot separate phasic from tonic pupil diameter changes [75]. Despite these inconsistencies, data from the present trial [31] was in line with a larger body of fMRI research that suggests intranasal oxytocin reduces amygdala activation. Future work should investigate both pupillometry and brain imaging data during the processing of more complex visual tasks.

There are some limitations worth noting. First, the study included a relatively low sample size. Underpowered studies are a considerable issue in biobehavioral oxytocin research as they are less likely to replicate [76]. However, the Bayes factors for the main effects and interactions indicated that the sample size was large enough to provide relative evidence for the alternative and null models. For a point of reference, underpowered studies tend to be associated with BFs $<3$ [61], which is much less than the reported Bayes factor of 15 . Second, the results can only be extrapolated to neurotypical male adults. Brain imaging research has demonstrated that oxytocin increases amygdala activity in females [77, 78], so it is unclear if this would also correspond with pupil dilation in females as well. Thus, future work would benefit from the recruitment of more representative populations. In addition, the present study is based on a partial reexamination of a published OT study $[31,41]$ with the addition of pupillometry data. Thus, the current results are not completely independent of previous findings. Third, as reported previously [31] there was no statistically significant main effect of task (i.e., the presentation of faces vs. shapes) on amygdala activity. It has been proposed that the amygdala facilitates the detection of behaviorally relevant stimuli, which incorporates but is not limited to emotion [79, 80]. As participants were instructed to answer questions regarding the visual characteristics of shapes, this was arguably a behaviorally relevant stimulus that was relevant to the participant's current goal, which may potentially explain the lack of difference between shapes and faces. Regardless of this lack of task effect, we still demonstrated a treatment effect as we observed that a low dose of intranasal oxytocin influenced both amygdala activity and pupil diameter compared to placebo and that pupil diameter was related to amygdala activity after a low dose of intranasal oxytocin. This may suggest that oxytocin may have more global effects on attention towards stimuli that are behaviorally relevant, regardless of emotional characteristics [64]. However, further research is needed to establish how and under which circumstances oxytocin impacts amygdala activation and to clarify the sources underlying the observed lack of a task effect. Our study is a first step toward a better understanding that needs to be replicated and extended.

In summary, this multi-dose and multi-delivery mode study of the effect of oxytocin in neurotypical males provides additional evidence that intranasal oxytocin exerts its neurobiological effects by increasing approach-related behaviors. Moreover, we provide new data consistent with the notion that the effects of oxytocin on the amygdala response to pupil dilation might involve a direct nose-to brain delivery pathway.

\section{ACKNOWLEDGEMENTS}

We thank Natalia Tesli, Claire Poppy, Hanne Smevik, Martin Tesli, Line Gundersen, Siren Tønnensen, Martina Lund, Eivind Bakken (NORMENT, KG Jebsen Center for Psychosis Research, Institute of Clinical Medicine, University of Oslo), Marianne Røine, Nils Meland, Claudia Grasnick, and Kristin A. Bakke (Smerud Medical Research International AS) for their contributions. We also thank medical staff from Oslo University Hospital and staff from the Oslo University Hospital Hormone laboratory for their assistance with the study. We are grateful to Sigma-Tau Industrie Farmaceutiche Riunite S.p.A. for their generous donation of the oxytocin used in the study.

\section{ADDITIONAL INFORMATION}

Supplementary Information accompanies this paper at (https://doi.org/10.1038/ s41386-018-0241-3).

Funding and disclosure: This study was supported by the Research Council of Norway and OptiNose AS (Grant no. BIA 219483) and an Excellence Grant for the Novo Nordisk Foundation (NNF16OC0019856). P.G.D. is an employee of OptiNose AS, Oslo, Norway and owns stock and stock options in OptiNose. O.A.A. has received speaker's honoraria from GSK, Lundbeck, and Otsuka for work not directly relevant to the submitted manuscript. R.A.M. is an employee of OptiNose US, Yardley, PA, USA and owns stock and stock options in OptiNose. K.T.S. is employed by Smerud Medical Research International AS, a CRO receiving fees for clinical trial services from OptiNose AS. The other authors declare no competing interests.

Publisher's note: Springer Nature remains neutral with regard to jurisdictional claims in published maps and institutional affiliations. 


\section{REFERENCES}

1. Davis MC, Lee J, Horan WP, Clarke AD, McGee MR, Green MF, et al. Effects of single dose intranasal oxytocin on social cognition in schizophrenia. Schizophr Res. 2013;147:393-7.

2. Domes G, Heinrichs M, Michel A, Berger C, Herpertz SC. Oxytocin improves "MindReading" in humans. Biol Psychiatry. 2007b;61:731-3.

3. Guastella AJ, Einfeld SL, Gray KM, Rinehart NJ, Tonge BJ, Lambert TJ, et al. Intranasal Oxytocin improves emotion recognition for youth with autism spectrum disorders. Biol Psychiatry. 2010;67:692-4.

4. Guastella AJ, Mitchell PB, Mathews F. Oxytocin enhances the encoding of positive social memories in humans. Biol Psychiatry. 2008b;64:256-8.

5. Guastella AJ, Mitchell PB, Dadds MR. Oxytocin increases gaze to the eye region of human faces. Biol Psychiatry. 2008a;63:3-5.

6. Yatawara C, Einfeld S, Hickie I, Davenport T, Guastella A. The effect of oxytocin nasal spray on social interaction deficits observed in young children with autism: a randomized clinical crossover trial. Mol Psychiatry. 2016;21:1225-31.

7. Quintana DS, Guastella AJ, Westlye LT, Andreassen OA. The promise and pitfalls of intranasally administering psychopharmacological agents for the treatment of psychiatric disorders. Mol Psychiatry. 2016a;21:29-38.

8. Alvares GA, Quintana DS, Whitehouse AJ. Beyond the hype and hope: critical considerations for intranasal oxytocin research in autism spectrum disorder. Autism Res. 2017;10:25-41.

9. Kosfeld M, Heinrichs M, Zak PJ, Fischbacher U, Fehr E. Oxytocin increases trust in humans. Nature. 2005;435:673-6.

10. MacDonald K, MacDonald TM. The peptide that binds: a systematic review of oxytocin and its prosocial effects in humans. Harv Rev Psychiatry. 2010;18:1-21.

11. Striepens N, Kendrick KM, Maier W, Hurlemann R. Prosocial effects of oxytocin and clinical evidence for its therapeutic potential. Front Neuroendocrinol. 2011;32:426-50.

12. Coghlan A. 'Cuddle chemical'eases symptoms of schizophrenia. New Sci. 2010;207:10

13. De Dreu CKW, Greer LL, Van Kleef GA, Shalvi S, Handgraaf MJJ. Oxytocin promotes human ethnocentrism. Proc Natl Acad Sci. 2011;108:1262-6.

14. Shamay-Tsoory SG, Fischer M, Dvash J, Harari H, Perach-Bloom N, Levkovitz Y. Intranasal administration of oxytocin increases envy and schadenfreude (gloating). Biol Psychiatry. 2009;66:864-70.

15. Shamay-Tsoory SG, Abu-Akel A. The social salience hypothesis of oxytocin. Biol Psychiatry. 2016;79:194-202.

16. Kemp AH, Guastella AJ. The role of oxytocin in human affect a novel hypothesis. Curr Dir Psychol Sci. 2011;20:222-31.

17. Carver CS, Harmon-Jones E. Anger is an approach-related affect: evidence and implications. Psychol Bull. 2009;135:183.

18. Prehn K, Heekeren HR, Van der Meer E. Influence of affective significance on different levels of processing using pupil dilation in an analogical reasoning task. Int J Psychophysiol. 2011;79:236-43.

19. Bradley MM, Miccoli L, Escrig MA, Lang PJ. The pupil as a measure of emotional arousal and autonomic activation. Psychophysiology. 2008;45:602-7.

20. Alnaes D, Sneve MH, Espeseth T, Endestad T, van de Pavert SHP, Laeng B. Pupil size signals mental effort deployed during multiple object tracking and predicts brain activity in the dorsal attention network and the locus coeruleus. J Vis. 2014;14:1-1

21. Karatekin C, Couperus JW, Marcus DJ. Attention allocation in the dual-task paradigm as measured through behavioral and psychophysiological responses. Psychophysiology. 2004:41:175-85.

22. Beatty J. Task-evoked pupillary responses, processing load, and the structure of processing resources. Psychol Bull. 1982;91:276.

23. Chatham $\mathrm{CH}$, Frank MJ, Munakata Y. Pupillometric and behavioral markers of a developmental shift in the temporal dynamics of cognitive control. Proc Natl Acad Sci. 2009;106:5529-33.

24. Leknes S, Wessberg J, Ellingsen DM, Chelnokova O, Olausson H, Laeng B. Oxytocin enhances pupil dilation and sensitivity to 'hidden' emotional expressions. Soc Cogn Affect Neurosci. 2012;8:741-9.

25. Prehn K, Kazzer $P$, Lischke A, Heinrichs M, Herpertz SC, Domes G. Effects of intranasal oxytocin on pupil dilation indicate increased salience of socioaffective stimuli. Psychophysiology. 2013;50:528-37.

26. Sansone GR, Gerdes CA, Steinman JL, Winslow JT, Ottenweller JE, Komisaruk BR, et al. Vaginocervical stimulation releases oxytocin within the spinal cord in rats. Neuroendocrinology. 2002;75:306-15.

27. Sansone GR, Komisaruk BR. Evidence that oxytocin is an endogenous stimulator of autonomic sympathetic preganglionics: the pupillary dilatation response to vaginocervical stimulation in the rat. Brain Res. 2001;898:265-71.

28. Domes G, Heinrichs M, Gläscher J, Büchel C, Braus DF, Herpertz SC. Oxytocin attenuates amygdala responses to emotional faces regardless of valence. Biol Psychiatry. 2007a;62:1187-90.
29. Labuschagne I, Phan KL, Wood A, Angstadt M, Chua P, Heinrichs M, et al. Oxytocin attenuates amygdala reactivity to fear in generalized social anxiety disorder. Neuropsychopharmacology. 2010;35:2403-13.

30. Petrovic $P$, Kalisch R, Singer T, Dolan RJ. Oxytocin attenuates affective evaluations of conditioned faces and amygdala activity. J Neurosci. 2008;28:6607-15.

31. Quintana DS, Westlye LT, Alnæs D, Rustan $\varnothing$, Kaufmann T, Smerud K, et al. Low dose intranasal oxytocin delivered with Breath Powered device dampens amygdala response to emotional stimuli: A peripheral effect-controlled withinsubjects randomized dose-response fMRI trial. Psychoneuroendocrinology. 2016b;69:180-8.

32. Ousdal O, Jensen J, Server A, Hariri A, Nakstad P, Andreassen O. The human amygdala is involved in general behavioral relevance detection: evidence from an event-related functional magnetic resonance imaging Go-NoGo task. Neuroscience. 2008;156:450-5.

33. Ebitz RB, Platt MM. An evolutionary perspective on the behavioral consequences of exogenous oxytocin application. Front Behav Neurosci. 2014;7:225.

34. Koikegami $\mathrm{H}$, Yoshida K. Pupillary dilatation induced by stimulation of amygdaloid nuclei. Psychiatry Clin Neurosci. 1953;7:109-26.

35. Ursin $\mathrm{H}$, Kaada BR. Functional localization within the amygdaloid complex in the cat. Electroencephalogr Clin Neurophysiol. 1960;12:1-20.

36. Horowitz J, Sears M. Neurohypophyseal peptides and the eye: use of synthetic analogs in analyzing effects on the pupil. Graefes Arch Clin Exp Ophthalmol. 1983;220:253-6.

37. Somppi S, Törnqvist $H$, Topál J, Koskela A, Hänninen L, Krause CM, et al. Nasal oxytocin treatment biases dogs' visual attention and emotional response toward positive human facial expressions. Front Psychol. 2017;8:1854.

38. Leng G, Ludwig M. Intranasal oxytocin: myths and delusions. Biol Psychiatry. 2016;79:243-50.

39. Striepens N, Kendrick KM, Hanking V, Landgraf R, Wüllner U, Maier W, et al. Elevated cerebrospinal fluid and blood concentrations of oxytocin following its intranasal administration in humans. Sci Rep. 2013;3:3440.

40. Quintana D, Westlye L, Hope S, Nærland T, Elvsåshagen T, Dørum E, et al. Dosedependent social-cognitive effects of intranasal oxytocin delivered with novel Breath Powered device in adults with autism spectrum disorder: a randomized placebo-controlled double-blind crossover trial. Transl Psychiatry. 2017;7:e1136.

41. Quintana DS, Westlye LT, Rustan ØG, Tesli N, Poppy CL, Smevik H, et al. Low dose oxytocin delivered intranasally with Breath Powered device affects socialcognitive behavior: a randomized 4-way crossover trial with nasal cavity dimension assessment. Transl Psychiatry. 2015b;5:1-9.

42. Wechsler D. Weschsler abbreviated scale of intelligence. San Antonio, TX: Psychological Corporation; 1999.

43. Lecrubier $Y$, Sheehan D, Weiller E, Amorim P, Bonora I, Harnett Sheehan $K$, et al. The Mini International Neuropsychiatric Interview (MINI). A short diagnostic structured interview: reliability and validity according to the CIDI. Eur Psychiatry. 1997;12:224-31.

44. Guastella AJ, Hickie IB, McGuinness MM, Otis M, Woods EA, Disinger HM, et al. Recommendations for the standardisation of oxytocin nasal administration and guidelines for its reporting in human research. Psychoneuroendocrinology. 2013;38:612-25.

45. Djupesland PG. Nasal drug delivery devices: characteristics and performance in a clinical perspective-a review. Drug Deliv Transl Res. 2012;3:42-62.

46. Djupesland PG, Mahmoud RA, Messina JC. Accessing the brain: the nose may know the way. J Cereb Blood Flow . 2013;33:793-4.

47. Cole P. The four components of the nasal valve. Am J Rhinol. 2003;17:107-10.

48. Lundqvist D, Flykt A, Öhman A. The Karolinska directed emotional faces (KDEF). CD ROM from Department of Clinical Neuroscience, Psychology section, Karolinska Institutet: Stockholm. 1998;91-630.

49. Fischl B, Salat DH, Busa E, Albert M, Dieterich M, Haselgrove C, et al. Whole brain segmentation: automated labeling of neuroanatomical structures in the human brain. Neuron. 2002;33:341-55.

50. Jenkinson $M$, Bannister $P$, Brady M, Smith S. Improved optimization for the robust and accurate linear registration and motion correction of brain images. Neuroimage. 2002;17:825-41.

51. Smith SM, Jenkinson M, Woolrich MW, Beckmann CF, Behrens TE, Johansen-Berg $\mathrm{H}$, et al. Advances in functional and structural MR image analysis and implementation as FSL. Neuroimage. 2004;23:S208-19.

52. Smith SM, Brady JM. SUSAN-A new approach to low level image processing. Int J Comput Vision. 1997;23:45-78.

53. Greve DN, Fischl B. Accurate and robust brain image alignment using boundarybased registration. Neuroimage. 2009;48:63-72.

54. Woolrich MW, Ripley BD, Brady M, Smith SM. Temporal autocorrelation in univariate linear modeling of FMRI data. Neuroimage. 2001;14:1370-86.

55. R Development Core Team. R: A language and environment for statistical computing. Vienna, Austria: R Foundation for Statistical Computing; 2014. 
56. Royall R. Statistical evidence: a likelihood paradigm. New York, USA: Routledge; 2017.

57. Quintana DS, Williams DR. Bayesian alternatives for common null-hypothesis significance tests in psychiatry: A non-technical guide using JASP. BMC Psychiatry. 2018;18:178.

58. Wagenmakers E-J, Marsman M, Jamil T, Ly A, Verhagen J, Love J, et al. Bayesian inference for psychology. Part I: Theoretical advantages and practical ramifications. Psychon Bull Rev. 2018;25:35-57.

59. Wetzels R, Wagenmakers E-J. A default Bayesian hypothesis test for correlations and partial correlations. Psychon Bull Rev. 2012;19:1057-64.

60. Jeffreys $\mathrm{H}$. The theory of probability. Oxford, UK: Oxford University Press; 1961.

61. Dienes Z. Using Bayes to get the most out of non-significant results. Front Psychol. 2014;5:1-17.

62. Quintana DS, Alvares GA, Hickie IB, Guastella AJ. Do delivery routes of intranasally administered oxytocin account for observed effects on social cognition and behavior? A two-level model. Neurosci Biobehav Rev. 2015a;49:182-92.

63. Barrett LF. Are emotions natural kinds? Perspect Psychol Sci. 2006;1:28-58.

64. Harari-Dahan O, Bernstein A. A general approach-avoidance hypothesis of oxytocin: accounting for social and non-social effects of oxytocin. Neurosci Biobehav Rev. 2014;47:506-19.

65. Bethlehem RAl, van Honk J, Auyeung B, Baron-Cohen S. Oxytocin, brain physiology, and functional connectivity: A review of intranasal oxytocin fMRI studies. Psychoneuroendocrinology. 2013;38:962-74.

66. Angrilli A, Mauri A, Palomba D, Flor H, Birbaumer N, Sartori G, et al. Startle reflex and emotion modulation impairment after a right amygdala lesion. Brain. 1996;119:1991-2004.

67. Phelps EA, O'Connor KJ, Gatenby JC, Gore JC, Grillon C, Davis M. Activation of the left amygdala to a cognitive representation of fear. Nat Neurosci. 2001;4:437.

68. Wright Cl, Fischer H, Whalen PJ, Mclnerney SC, Shin LM, Rauch SL. Differential prefrontal cortex and amygdala habituation to repeatedly presented emotional stimuli. Neuroreport. 2001;12:379-83.
69. Samson WK. Oxytocin redux. Am J Physiol-Regulat Integrat Comp Physiol: ajpregu. 00307.02016; 2016.

70. Stoehr JD, Cramer CP, North WG. Oxytocin and vasopressin hexapeptide frag ments have opposing influences on conditioned freezing behavior. Psychoneuroendocrinology. 1992;17:267-71.

71. Liberzon I, Trujillo KA, Akil H, Young EA. Motivational properties of oxytocin in the conditioned place preference paradigm. Neuropsychopharmacology. 1997;17:353-9.

72. Yang J, Yang Y, Chen J-M, Liu W-Y, Wang C-H, Lin B-C. Central oxytocin enhances antinociception in the rat. Peptides. 2007;28:1113-9.

73. Singer T, Snozzi R, Bird G, Petrovic P, Silani G, Heinrichs M, et al. Effects of oxytocin and prosocial behavior on brain responses to direct and vicariously experienced pain. Emotion. 2008;8:781.

74. Gross J, De Dreu CK. Oxytocin conditions trait-based rule adherence. Soc Cogn Affect Neurosci. 2017;12:427-35.

75. Gilzenrat MS, Nieuwenhuis S, Jepma M, Cohen JD. Pupil diameter tracks changes in control state predicted by the adaptive gain theory of locus coeruleus function. Cogn Affect Behav Neurosci. 2010;10:252-69.

76. Walum H, Waldman ID, Young L. Statistical and methodological considerations for the interpretation of intranasal oxytocin studies. Biol Psychiatry. 2016;79:251-7.

77. Domes G, Lischke A, Berger C, Grossmann A, Hauenstein K, Heinrichs M, et al Effects of intranasal oxytocin on emotional face processing in women. Psychoneuroendocrinology. 2010;35:83-93.

78. Lischke A, Gamer M, Berger C, Grossmann A, Hauenstein K, Heinrichs M, et al. Oxytocin increases amygdala reactivity to threatening scenes in females. Psychoneuroendocrinology. 2012;37:1431-8.

79. Ousdal OT, Reckless GE, Server A, Andreassen OA, Jensen J. Effect of relevance on amygdala activation and association with the ventral striatum. Neuroimage. 2012;62:95-101.

80. Sander D, Grafman J, Zalla T. The human amygdala: an evolved system for relevance detection. Rev Neurosci. 2003;14:303-16. 